\title{
MOMENT LYAPUNOV EXPONENT OF DELAY DIFFERENTIAL EQUATIONS
}

\author{
M. S. FOFANA
}

Received 20 February 2001 and in revised form 9 September 2001

\begin{abstract}
The aim of this paper is to establish a connecting thread through the probabilistic concepts of $p$ th-moment Lyapunov exponents, the integral averaging method, and Hale's reduction approach for delay dynamical systems. We demonstrate this connection by studying the stability of perturbed deterministic and stochastic differential equations with fixed time delays in the displacement and derivative functions. Conditions guaranteeing stable and unstable solution response are derived. It is felt that the connecting thread provides a unified framework for the stability study of delay differential equations in the deterministic and stochastic sense.
\end{abstract}

2000 Mathematics Subject Classification: 92C20.

1. Introduction. Concepts of $p$ th-moment Lyapunov exponent have been employed in the study of stability behaviour of structural systems with stochastically perturbed excitations, where governing equations for single-degree-of-freedom systems are typically of nonlinear stochastic ordinary differential equations (ODEs) of the form

$$
\ddot{x}+\left[2 \delta_{0} \omega_{0}+\varepsilon^{1 / 2} \sigma_{0} \xi(t)\right] \dot{x}+\omega_{0}^{2}\left[1+\varepsilon^{1 / 2} \sigma_{1} \gamma(t)\right] x+\varepsilon \sigma_{3} x^{3}=0,
$$

where $\delta_{0}$ and $\omega_{0}$ represent the damping ratio and the undamped natural frequency of the excitations, respectively. The processes $\xi(t)$ and $\gamma(t)$ are independent processes which are typically stationary with zero mean values; $\sigma_{0}, \sigma_{1}$ are the noise intensities of the processes, $\varepsilon$ is a small parameter that takes values between 0 and 1 , while $\sigma_{3}$ is a real constant denoting the nonlinear perturbation. The name moment Lyapunov exponent comes from the connection of $p$ th-moment stability, which we denote here as $\mathfrak{I}(p)$, and the sample stability or Lyapunov exponent of stochastic solutions which is denoted by $\pi_{\exp }$. The concepts have become the most attractive aspects for the study of stability behaviour of stochastic dynamical systems. The parameter $p \in \mathfrak{R}$ of moment exponent is a unique number and it stands for stability index of the solutions.

To define the concepts of $p$ th-moment Lyapunov exponent, we consider the special linearized case of (1.1), written by means of the transformation $x=x_{1}, \dot{x}=x_{2}$, and along with the assumed equilibrium conditions $x_{1}(0)=x_{0}, x_{2}(0)=v_{0}$, as form of a pair of Itô ODEs, namely

$$
\begin{gathered}
d x_{1}(t)=x_{2} d t \\
d x_{2}(t)=-\left\{\omega_{0}^{2} x_{1}+\left(2 \delta_{0} \omega_{0}-\frac{\varepsilon \sigma_{0}^{2}}{2}\right) x_{2}\right\} d t-\varepsilon^{12} \sigma_{0} x_{2} d W(t),
\end{gathered}
$$


where $W(t)$ is a unit Wiener process. By the multiplicative ergodic theorem of Oseledec [7], the Lyapunov exponent and the $p$ th-moment exponent of solutions to (1.2) and (1.3) are defined, respectively, as

$$
\begin{aligned}
& \pi_{\exp }=\lim _{t \rightarrow \infty} t^{-1}\left\{\log \left\{\left|x_{1}\left(t, x_{0}, v_{0}\right)\right|^{2}+\left|x_{2}\left(t, x_{0}, v_{0}\right)\right|^{2}\right\}^{1 / 2}\right\}, \\
& \mathfrak{J}(p)=\lim _{t \rightarrow \infty} \log E\left[\left\{\left\{\left|x_{1}\left(t, x_{0}, v_{0}\right)\right|^{2}+\left|x_{2}\left(t, x_{0}, v_{0}\right)\right|^{2}\right\}^{1 / 2}\right\}^{p}\right],
\end{aligned}
$$

in which $E[\cdot]$ stands for the expected value of the quantity within the square bracket. In (1.5), if the exponent $\mathfrak{I}(p)<0$, then by definition $E\left[\{\cdot\}^{p}\right] \rightarrow 0$ as $t \rightarrow \infty$, and thus we can say that the solution response to (1.2) and (1.3) is $p$ th-moment stability in the almost-sure sense. On the contrary, that is, for $\mathfrak{I}(p)>0$, the expectation $E\left[\{\cdot\}^{p}\right] \neq 0$ as $t \rightarrow \infty$, and thus $p$ th-moment instability will occur in the almost-sure sense. The values of $\pi_{\exp }$ and $\mathfrak{J}(p)$ are real and deterministic in nature as long as the random system is ergodic. By the $p$ th-moment Lyapunov exponent of a stochastic dynamical system, we mean that there is a $p$ th-moment stability of the corresponding random linear solutions of the system in the almost-sure sense. This means that, among an $n$ number of exponents, if the maximal Lyapunov exponent is negative (i.e., $\pi_{\exp }<0$ ), the random system is almost-surely stable for small values of the stability index $p$. However, in this situation $p$ th-moment grows exponentially for large values of $p$, and thereby indicating that $p$ th-moment response of the system is unstable. A remarkable observation one can infer from this, is that although at an exponential rate we may have $\pi_{\exp }<0$, thereby resulting to stability of the solution response in the almost-sure sense, yet for large values of $p$ there is small probability of chance that the response would be large. Corresponding expected values for this rare event are indeed also large, and it is conclusive to say that $p$ th-moment exponent of the system is unstable. Opposite of this situation is when the Lyapunov exponent is positive (i.e., $\pi_{\exp }>0$ ) and the system is almost-surely unstable.

The values for the stability index $p$ are usually determined by the solutions of $\mathfrak{I}(p)=0$, and these values are dependent upon the dimension of the random dynamical system. It has been shown by Baxendale [3] that the corresponding values of $p$ when $\mathfrak{T}(p)=0$ equal the negative of the dimension of the system. For example a system with a dimension $n$, if for a solution of $\mathfrak{I}(p)=0$, we have $p=p_{1} \neq 0$, then we can write $p_{1}=-n$. At such a point, $p=p_{1}$, there is an expectation that the sign of the maximal value of the Lyapunov exponent $\pi_{\exp }$ will change from negative to positive, and thus a change in character of the corresponding probability density function will occur as well. Traditionally, there is an obvious computational difficulty if one wishes to determine $\mathfrak{I}(p)$ for many arbitrary values of $p$. Efforts by Arnold et al. [1, 2] and many others in the stochastic community, led to the fact that the $p$ thmoment Lyapunov exponent $\mathfrak{I}(p): \mathfrak{R} \rightarrow \mathfrak{R}$ is a convex and analytic function in $p$ in such a way that the expression $\mathfrak{I}(p) / p$ increases and $\mathfrak{I}(p)=\{0\}_{p=p_{1}}$. Thus by taking the asymptotic expansion of $\mathfrak{J}(p)$ for $p=p_{1}$ near zero, and bearing in mind that $(d \mathfrak{J} / d p)(0)=\pi_{\exp }, \mathfrak{J}(0)=0$, we have

$$
\mathfrak{J}(p)=p \pi_{\exp }+(2 !)^{-1} p^{2} \frac{d^{2} \mathfrak{I}}{d p^{2}}(0)+(3 !)^{(-1)} p^{3} \frac{d^{3} \mathfrak{J}}{d p^{3}}(0)+O\left(p^{n}\right) .
$$


This asymptotic connection indeed brought about the concepts of large deviations of linear random dynamical systems in the stability study of solution responses. The asymptotic expansion has been employed in the literature to determine rare situations where negative maximal Lyapunov exponent, $p$ th-moment solution response grow exponentially for large values of $p$.

Another attractive aspect of $p$ th-moment Lyapunov exponent is its further connection with the integral stochastic averaging method. It was Has'minskiı [5] and Stratonovich [9] who developed formulas for such a connection, and the formulas were based upon the ideas of Bogoliubov and Mitropolski [6] in the context of deterministic averaging method. When the integral stochastic averaging method is applied to equations of the form (1.2) and (1.3), it produces Markovian solutions to the nonlinear systems in terms of amplitude and phase relations, which can be written either in the sense of Stratonovich or Itô using stochastic differential rules. The rules treat differentiation of functions mapped originally from a logarithmic polar transformation of the vector state variables, which depend on the corresponding Itô equations. The connection between these probabilistic concepts and the stability study of delay differential equations (DDEs), by means of Andronov-Hopf bifurcation and centre manifold, is the focus of this investigation. In pursuing this, we expect to provide a unified framework for the study of stability of DDEs with deterministic and stochastic perturbations. First, we will use the Andronov-Hopf bifurcation and centre manifold according to Hale [4] to reduce the infinite-dimensional character of the DDEs into family of ODEs in the space $C:=C\left([-\tau, 0], \mathfrak{R}^{2}\right)$. The integral averaging of the ODEs produces averaged equations in terms of amplitude and phase relations. From the averaged equations, explicit expressions for the occurrence of stable and unstable solution responses will be derived using $p$ th-moment Lyapunov exponent.

2. Hale's reduction approach (see [4]). Let $L=L\left(x_{t}(\theta), \mu\right): C x \mathfrak{R} \rightarrow \mathfrak{R}^{n}$ and $\Delta f=$ $\Delta f\left(x_{t}(\theta), \mu, \varepsilon\right): \mathfrak{R} \times C \rightarrow \mathfrak{R}^{n}$ denote accordingly the linear and nonlinear functional mappings depending upon a parameter $\mu$ and the state variable $x_{t}(\theta)$. The variable $x_{t}(\theta)$ is contained in $C:=C\left([-\tau, 0], \Re^{n}\right)$, the Banach space of all continuous functions equipped with the usual supremum norm $\|\cdot\|$ in $C$ and vector norm $|\cdot|$ in $\mathfrak{R}^{n} ; x_{t}(\theta) \in$ $C$ represents the past history solution of a delay differential equation of the form

$$
\dot{x}(t)=L\left(x_{t}(\theta), \mu\right) x_{t}(\theta)+\varepsilon \triangle f\left(x_{t}(\theta), \mu, \varepsilon\right), \quad 0 \leq \varepsilon \ll 1,
$$

whose trajectory will coincide with the solution of the future state variable $x(t) \in \mathfrak{R}^{n}$ through the definition $x_{t}(\theta)=x(t+\theta),-\tau \leq \theta \leq 0, t \geq 0$, where $\tau>0$ is a fixed time delay. For a given initial continuously differential function $\phi(\theta) \in C$ having supremum norm $\|\phi(\theta)\|=\sup _{-\tau \leq \theta \leq 0}|\phi(\theta)|$, we can say that the function $x(\phi(0), t, \mu) \in \mathfrak{R}^{n}$ through $\phi(\theta)$ with initial value $\phi(0)$ at zero is the solution to (2.1) if and only if $x_{t}(\phi(\theta), \mu) \in C$ satisfies the variation of constants-integral equation

$$
x_{t}(\phi(\theta), \mu)=J(t, \mu) \phi(\theta)+\varepsilon \int_{0}^{t} J((t-\xi), \mu) X_{0}(\theta) \triangle f(\phi(\theta), \mu, \varepsilon) d \xi,
$$

where the element $X_{0}(\theta)$ is $n \times n$ matrix function defined as $X_{0}(\theta)=0,-\tau \leq \theta<0$ and $X_{0}(0)=I, \theta=0$, and $I$ is the identity matrix. In this integral equation, $J(t, \mu)$ is 
defined for $t, \mu \geq 0$, and it is a bounded linear operator with infinitesimal generator $A(\theta, \mu) \in C$ described via the linear delay equation when $\varepsilon=0$ in (2.1), namely

$$
\dot{x}(t)=L\left(x_{t}(\theta), \mu\right) x_{t}(\theta), \quad \phi(\theta)=x(\theta), \quad-\tau \leq \theta \leq 0,
$$

in which for $\phi(\theta) \in C$, we have $D(A(\theta, \mu))=\phi(\theta) \in C: \dot{\phi}(\theta) \in C, \dot{\phi}(\theta)=L=$ $L_{-\tau}(\phi(-\tau), \mu)+L_{0}(\phi(0), \mu), \theta=0$ and $A(\theta, \mu) \phi(\theta)=\dot{\phi}(\theta),-\tau \leq \theta<0$, where $L_{-\tau}(\phi(-\tau), \mu)$ and $L_{0}(\phi(0), \mu)$ are $n \times n$ matrices. For a given function $\eta(\theta, \mu)$ : $[-\tau, 0] \rightarrow \mathfrak{R}^{n}$ of bounded variation in $[-\tau, 0]$, described by $\eta(\theta, \mu)=L_{-\tau}((-\tau), \mu)$ when $\theta=-\tau, 0,-\tau<\theta<0$ and $L_{0}((0), \mu)$, and $\theta=0$, we have the representation $L\left(x_{t}(\theta), \mu\right) x_{t}(\theta)=\int_{-\tau}^{0}[d \eta(\theta, \mu)] x_{t}(\theta) . J(t, \mu)$ is a semigroup, that is, for $t, \varsigma \geq 0$, we have $J(t, \mu), J(t, \mu), J(\varsigma, \mu)=J(t+\varsigma, \mu)$, and furthermore $J(0, \mu)=I$ for $t=0$. It maps $C$ into itself, namely $J(t, \mu): C x \Re \rightarrow C$, or equivalently carries the solution state evolution of $x_{t}(\phi(\theta), \mu)$ in $C$ onto $\mathfrak{R}^{n}$ by the relation $x_{t}(\phi(\theta), \mu)=J(t, \mu) \phi(\theta)$, where $J(t, \mu) \phi(\theta)$ is the solution operator of (2.3). For $\phi(\theta) \in D(A(\theta, \mu))$, the solution operator $J(t, \mu) \phi(\theta)$ is differentiable and there is the differential equation

$$
\frac{d}{d t}[J(t, \mu) \phi(\theta)]=A(\theta, \mu)[J(t, \mu) \phi(\theta)]=J(t, \mu)[A(\theta, \mu) \phi(\theta)] .
$$

Then the action of $J(t, \mu)$ on the function $\phi(\theta)$ is described by $J(t, \mu) \phi(\theta)=$ $\phi(0) e^{B(t+\theta)},-\tau \leq \theta \leq 0, t \geq 0$, while the action of $(A(\theta, \mu))$ on $\phi(\theta)$ is given by $A(\theta, \mu) \phi(\theta)=\phi(\theta) B,-\tau \leq \theta \leq 0 . B \in C$ is an $n \times n$ constant matrix whose elements are the eigenvalues with zero real parts of the transcendental characteristic equation

$$
\Delta(\lambda, \mu):=\operatorname{det}\left\{\lambda I-\int_{-\tau}^{0}[\operatorname{dn}(s, \mu)] e^{\lambda \theta}\right\}=0,
$$

associated with (2.3). The properties of $J(t, \mu)$ and the unique correspondence between $J(t, \mu)$ and its generator $A(\theta, \mu)$ ensure that the spectra sets $\sigma(J(t, \mu))$ of $J(t, \mu)$ and $\sigma(A(\theta, \mu))$ of $A(\theta, \mu) \in C$ are the point spectra of the finite type, and they consist of those eigenvalues that satisfy (2.5). This equation $\Delta(\lambda, \mu)=0$ in (2.5) has infinite-dimensional eigenvalues, and among them we will assume that the parameter $\mu$ varies near $\mu_{c}$, and there exists a finite number of eigenvalues of the form $\wedge(\lambda, \mu)=\left\{\lambda_{1}, \lambda_{2}, \lambda_{3}, \ldots, \lambda_{k}\right\}, k=1,2, \ldots, n$. Furthermore, we assume that these eigenvalues are exactly the point spectrum of $J(t, \mu)$ and its generator $A(\theta, \mu)$, and they have positive real and complex conjugate parts. All other eigenvalues of $\Delta(\lambda, \mu)=0$ are assumed to have negative real parts.

With the above assumptions, we decompose $C$ into the generalized eigenspace $P=P(\lambda, \mu) \in C$ and complementary subspace $Q=Q(\lambda, \mu) \in C$ as $C=P \oplus Q$ by all the eigenvalues of (2.5), where $P$ is associated with the $k$-dimensional eigenvalues of $\wedge(\lambda, \mu)$ and $Q$ corresponds to all the remaining infinite-dimensional eigenvalues of $\Delta(\lambda, \mu)=0$ with negative real parts. The subspaces $P, Q$ are disjoint and invariant under $J(t, \mu)$ and $A(\theta, \mu)$. This way, if for example $\phi(\theta) \in P$, then a solution $x(\phi(0), t, \mu)$ defined on $[-\tau, \infty)$ has a backward extension on $(-\infty,-\tau]$, and indeed $x_{t}(\phi(\theta), \mu)$ remains in $P$ for all values of time $t \in(-\infty, \infty)$. The decomposition $C=P \oplus Q$ by $\Delta(\lambda, \mu)=0$ yields that the elements $\phi(\theta)$ and $X_{0}(\theta)$ of the integral equation (2.2) in $C$ can have the unique representations $\phi(\theta)=\phi^{P}(\theta)+\phi^{Q}(\theta)$ and 
$X_{0}(\theta)=X_{0}^{P}(\theta)+X_{0}^{Q}(\theta)$ where the superscripts denote their respective projections onto $P$ and $Q$. Also, for the integral solution $x_{t}(\phi(\theta), \mu)$ in $C$, we have the representation $x_{t}(\phi(\theta), \mu)=x_{t}^{P}(\phi(\theta), \mu)+x_{t}^{Q}(\phi(\theta), \mu)$ where the projections $x_{t}^{P}(\phi(\theta), \mu)$ and $x_{t}^{Q}(\phi(\theta), \mu)$ are given by

$$
\begin{aligned}
& x_{t}^{P}(\phi(\theta), \mu)=J(t, \mu) \phi^{P}(\theta)+\varepsilon \int_{0}^{t} J((t-\xi), \mu) X_{0}^{P}(\theta) \triangle f(\phi(\theta), \mu, \varepsilon) d \xi, \quad t \in(-\infty, \infty), \\
& x_{t}^{Q}(\phi(\theta), \mu)=J(t, \mu) \phi^{Q}(\theta)+\varepsilon \int_{0}^{t} J((t-\xi), \mu) X_{0}^{Q}(\theta) \triangle f(\phi(\theta), \mu, \varepsilon) d \xi, \quad t \in[0, \infty),
\end{aligned}
$$

and the elements in these equations are explicitly defined by

$$
\begin{gathered}
\phi(\theta) \in C \mid \phi^{P}(\theta)=\phi(\theta)-\phi^{Q}(\theta), \quad \phi^{P}(\theta)=\Phi(\theta) b, \quad b=\left(\bar{\Psi}(s), \phi^{P}(\theta)\right), \\
X_{0}(\theta) \in C \mid X_{0}^{P}(\theta)=X_{0}(\theta)-X_{0}^{Q}(\theta), \quad X_{0}(\theta):=\Phi(\theta) \bar{\Psi}(0), \quad-\tau \leq \theta \leq 0 .
\end{gathered}
$$

Furthermore, it is known that the restriction of the semigroup $J(t, \mu)$ to the subspace $P$ constitutes a generalized centre manifold $M_{\mu}=M_{\mu}(A(\theta, \mu)) \in C\left([-\tau, 0], \mathfrak{R}^{n}\right)$. This centre manifold is tangent to the subspace $P$ at a point where $P$ and $Q$ are orthogonal. On this centre manifold, long-term qualitative behaviour of the original nonlinear delay equation (2.1) can be well approximated by the behaviour of the finite-dimensional ODEs restricted to the point spectrum of $J(t, \mu)$ and $A(\theta, \mu)$, or equivalently to the eigenvalues of $\wedge(\lambda, \mu)=\left\{\lambda_{1}, \lambda_{2}, \lambda_{3}, \ldots, \lambda_{k}\right\}$. The ODEs come from the variation of constant integral equation $x_{t}^{P}(\phi(\theta), \mu)$ of the generalized eigenspace $P \in C$.

The function $\Phi(\theta)$ in (2.7) is a basis for $P \in C$, and its elements are the linearly independent solutions of (2.3) for which $\wedge(\lambda, \mu)=\left\{\lambda_{1}, \lambda_{2}, \lambda_{3}, \ldots, \lambda_{k}\right\}$ are eigenvalues of (2.5). That is, for all $\lambda_{k}$ of $\wedge(\lambda, \mu)$, we have $\phi_{k}(\theta)=e^{\lambda_{k} \theta}$ where we denote $\Phi(\theta)=\left[\phi_{1}(\theta), \phi_{2}(\theta), \ldots, \phi_{k}(\theta)\right] \in C$. Also, corresponding to these finite-dimensional eigenvalues is the $k \times k$ constant matrix $B$ in $C$. This matrix $B$ is usually the Jordancanonical form whose elements are the eigenvalues of $\Delta(\lambda, \mu)=0$ with zero real parts, and they can be determined by means of the definition $A(\theta, \mu) \Phi(\theta)=\Phi(\theta) B$ such that the identity $\Phi(\theta)=\Phi(0) e^{B \theta}$ for $-\tau \leq \theta \leq 0$ holds. $\bar{\Psi}(0)$ is the normalized basis of $\Psi(s)=\left[\psi_{1}(s), \psi_{2}(s), \ldots, \psi_{k}(s)\right] \in \hat{C}, \psi_{k}(s)=e^{-\lambda_{k} s}$ at $s=0$ for the generalized eigenspace $\hat{P}=\hat{P}(\lambda, \mu) \in \hat{C}$ corresponding to the functional delay differential equations

$$
\begin{gathered}
\dot{u}_{\hat{t}}(s)=\hat{L}\left(u_{\hat{t}}(s), \mu\right) u_{\hat{t}}(s), \quad u_{\hat{t}}(s) \in \hat{C}:=\hat{C}\left([0, \tau], \mathfrak{R}^{n}\right), \quad \hat{L}\left(u_{\hat{t}}(s), \mu\right): \hat{C} x \mathfrak{R} \rightarrow \mathfrak{R}^{n}, \\
\hat{L}\left(u_{\hat{t}}(s), \mu\right) u_{\hat{t}}(s)=-\int_{-\tau}^{0}[d \hat{\eta}(s, \mu)] u_{\hat{t}}(s), \quad u_{\hat{t}}(s)=\psi(s), \\
u_{\hat{t}}(s)=u(-\hat{t}+s), \quad 0 \leq s \leq \tau, \hat{t} \in[0, \infty),
\end{gathered}
$$

formally adjoint to (2.3) with respect to the bilinear relation

$$
\begin{gathered}
\left(\psi_{j}(s), \phi_{k}(\theta)\right)=\left(\psi_{j}(0), \phi_{k}(0)\right)-\int_{-\tau}^{0} \int_{0}^{\theta} \psi_{j}(\xi-s)[d \eta(\theta, \mu)] \phi_{k}(\xi) d \xi \\
\phi_{k}(\theta) \in C, \psi_{j}(s) \in \hat{C}, j, k=1,2,3, \ldots, n,
\end{gathered}
$$


in $C \times \hat{C}$. The normalized basis $\bar{\Psi}(s)$ is determined by computing $\bar{\Psi}(s)=(\Psi(s)$, $\Phi(\theta))^{-1} \Psi(s)$ where $(\Psi(s), \Phi(\theta))$ is a $k \times k$ inner product matrix with the bilinear relation in (2.9) as elements. That is, for $\phi_{k}(\theta) \in C, \psi_{j}(s) \in \hat{C}$, we have $(\Psi(s), \Phi(\theta))=$ $\left(\psi_{j}(s), \phi_{k}(\theta)\right), j, k=1,2, \ldots, n$, which usually produces a nonsingular value after the substitution of its elements into (2.9). That is, $(\Psi(s), \Phi(\theta)) \neq I$. However, with the normalized basis $\bar{\Psi}(s) \in \hat{C}$ for $\hat{P} \in \hat{C}$, we can see that the substitution of the elements $\left(\bar{\psi}_{j}(s), \phi_{k}(\theta)\right)$ of the new inner product $(\bar{\Psi}(s), \Phi(\theta))$ produces the identity matrix, $(\bar{\Psi}(s), \Phi(\theta))=I$. Next interest is to determine the exponential estimates of the projected solutions $x_{t}^{P}(\phi(\theta), \mu)$ and $x_{t}^{Q}(\phi(\theta), \mu)$, and for this purpose we need the following theorem.

THEOREM 2.1. For any real number, say $\mu$, let $\wedge(\lambda, \mu)=\{\lambda \in A(\theta, \mu) \mid \Delta(\lambda, \mu)=$ $0, \mathfrak{R} e \lambda>\mu\}$ be the point spectral set of the finite type and has eigenvalues satisfying the characteristic equation (2.5). If $C$ is decomposed into the generalized eigenspace $P$ and the complementary subspace $Q$ by all the eigenvalues of $\Delta(\lambda, \mu)=0$ as $C=P \oplus Q$. Then, for any $\phi(\theta) \in C$ with the representation $\phi(\theta)=\phi^{P}(\theta)+\phi^{Q}(\theta)$ there exist positive constants $\delta$ and $\kappa=\kappa(\delta)$ such that the following inequalities hold:

$$
\begin{aligned}
& \left\|J(t, \mu) \phi^{P}(\theta)\right\| \leq \kappa e^{(\mu+\delta) t}\left\|\phi^{P}(\theta)\right\|, \quad t \leq 0, \\
& \left\|J(t, \mu) \phi^{Q}(\theta)\right\| \leq \kappa e^{-(\mu+\delta) t}\left\|\phi^{Q}(\theta)\right\|, \quad t \geq 0 .
\end{aligned}
$$

REMARK 2.2. A proof of the above theorem is given in the classical book of Hale [4]. By means of (2.10), numerous autonomous and nonautonomous time delay perturbations have been estimated. Here, we exploit the insights that emerged from the proof of these inequalities. Namely, any projected solution of equations (2.3) onto $P \in C$ is bounded as $t \rightarrow-\infty$, and unbounded when $t \rightarrow \infty$, while a solution projection onto $Q \in C$ will remain bounded for all values of $t \geq 0$. In particular, having $\phi^{Q}(\theta)=\phi(\theta)-\phi^{P}(\theta)$ and $\phi^{P}(\theta)=\Phi(\theta) b$ such that $J(t, \mu) \Phi(\theta)=\Phi(0) e^{B(t+\theta)}$, we have from the first inequality that $\left\|J(t, \mu) \phi^{P}(\theta)\right\| \rightarrow 0$, as $t \rightarrow-\infty$, and while the second inequality will yield $\left\|J(t, \mu) \phi^{Q}(\theta)\right\| \rightarrow 0$, as $t \rightarrow \infty$. Also, by means of the first inequality in (2.10), it can be shown that the exponential estimate for $x_{t}^{P}(\phi(\theta), \mu)$, $t \in(-\infty, \infty)$ defined on $P$ are bounded as $t \rightarrow-\infty$ and unbounded when $t \rightarrow \infty$. The latter is indeed the situation which produces the corresponding ODEs on the centre manifold.

Hence, for the estimation of the nonlinear variation of constant integral solutions $x_{t}^{P}(\phi(\theta), \mu), x_{t}^{Q}(\phi(\theta), \mu)$ in (2.6) and (2.7) we have from the second inequality the following:

$$
\begin{aligned}
\| J(t, \mu) & \phi^{Q}(\theta) \| \\
& =\left\|x_{t}^{Q}(\phi(\theta), \mu)-\varepsilon \int_{0}^{t} J((t-\xi), \mu) X_{0}^{Q}(\theta) \triangle f(\phi(\theta), \mu, \varepsilon) d \xi\right\|, \quad t \in[0, \infty), \\
X_{0}^{Q}(\theta) & =\left\{X_{0}(\theta)-X_{0}^{P}(\theta) \mid X_{0}(\theta):=\Phi(\theta) \bar{\Psi}(0)\right\}, \\
\phi^{Q}(\theta) & =\phi(\theta)-\phi^{P}(\theta),
\end{aligned}
$$


where the left-hand side of this equation exponentially converges to zero as $t \rightarrow \infty$, namely

$$
\begin{aligned}
\left\|J(t, \mu) \phi^{Q}(\theta)\right\| \equiv & \left\|J(t, \mu)\left\{\phi(\theta)-\phi^{P}(\theta)\right\}\right\|=\left\|J(t, \mu) \phi(\theta)-\Phi(0) e^{B t} b\right\| \\
\equiv & \left\|J(t, \mu) \Phi(0) e^{B t} b-\Phi(0) e^{B t} b\right\| \longrightarrow 0, \\
& t \rightarrow \infty, \quad J(0, \mu)=I, \quad b:=\left(\bar{\Psi}(s), \phi^{P}(\theta)\right) .
\end{aligned}
$$

In a similar way, the first inequality produces $\left\|x_{t}^{P}(\phi(\theta), \mu)\right\| \rightarrow 0$ as $t \rightarrow-\infty$. As firstorder approximation, one can accept these convergencies, and then describe the longterm behaviour of the original delay differential equations (2.1) with the corresponding set of ODEs for $x_{t}^{P}(\phi(\theta), \mu),-\tau \leq \theta \leq 0, t \in[0, \infty)$ on the generalized centre manifold $M_{\mu} \in C$.

Therefore, on the centre manifold $M_{\mu} \in C$, we have the corresponding solution of (2.1) as

$$
\begin{gathered}
M_{\mu}=x_{t}^{P}(\phi(\theta), \mu) \in C, \quad x_{t}^{P}(\phi(\theta), \mu)=\Phi(\theta) z(t)+x_{t}^{Q}(\phi(\theta), \mu), \\
z(t)=\left(\bar{\Psi}(s), \phi^{P}(0)\right), z(t) \in \mathfrak{R}^{k} .
\end{gathered}
$$

Since we know that the exponential estimate for $x_{t}^{Q}(\phi(\theta), \mu)$ in the complementary subspace $Q$ is zero, then the change of variables $x_{t}^{P}(\phi(\theta), \mu)=\Phi(\theta) z(t),-\tau \leq \theta \leq 0$, and their differentiation with respect to time $t$ produces

$$
\begin{aligned}
\Phi(\theta) \dot{z}(t) & =\dot{x}_{t}^{P}(\phi(\theta), \mu) \\
& =\frac{d}{d t}\left\{J(t, \mu) \phi^{P}(\theta)+\varepsilon \int_{0}^{t} J((t-\xi), \mu) X_{0}^{P}(\theta) \triangle f(\phi(\theta), \mu, \varepsilon) d \xi\right\},
\end{aligned}
$$

where the substitution of $X_{0}^{P}(\theta):=\Phi(\theta) \bar{\Psi}(0)$ into this equation gives the $k$-dimensional ODEs

$$
\dot{z}(t)=B z+\bar{\Psi}(0) \triangle f(\Phi(\theta) z(t), \mu, \varepsilon), \quad z(t) \in \mathfrak{R}^{k}, t \in[-\tau, \infty),
$$

and $B$ is a $k \times k$ matrix. We now illustrate these ideas by two examples with fixed time delays.

3. An illustrative example I. The specific single-degree-of-freedom dynamical system considered is represented by the second-order DDEs of the form

$$
\begin{gathered}
\dot{x}_{1}(t)=x_{2}, \\
\dot{x}_{2}(t)=-\omega_{0}^{2} x_{1}-2 \delta_{0} \omega_{0} x_{2}-\mu \omega_{0}^{2}\left\{x_{1}\left(t-\tau_{1}\right)+\delta_{1} x_{2}\left(t-\tau_{2}\right)\right\}-\varepsilon^{1 / 2} \sigma_{0} \xi(t) x_{2},
\end{gathered}
$$

in $C:=C\left([-\tau, 0], \mathfrak{R}^{2}\right)$, where $\tau_{2} \leq \tau \leq \tau_{1}$. All the parameters contained in these equations are real and $\mu$ is the selected bifurcation parameter, which is set to vary by $\varepsilon \tilde{\mu}$ in the neighborhood of some critical value $\mu_{c}$, namely $\mu=\mu_{c}+\varepsilon \tilde{\mu} . \tau_{1}, \tau_{2}$ are the respective time delays in the restoring and damping forces. Equations of the form (3.1) have been encountered in the active controlling of structural systems with earthquake excitations [8], where the parameter $\mu$ often stand for the gain of the delayed forces. 
We study the stability of (3.1) by examining the eigenvalues of the transcendental characteristic equation

$$
\Delta(\lambda, \mu):=\lambda^{2}+\left(2 \delta_{0}+\mu \omega_{0} \delta_{1} e^{-\lambda \tau_{2}}\right) \omega_{0} \lambda+\omega_{0}^{2}(1+\mu) e^{-\lambda \tau_{1}}=0,
$$

which is associated with the linearized part when $\sigma_{0}=0$. Along with the linear DDEs in (3.1), we consider their corresponding adjoint form

$$
\begin{aligned}
& \dot{u}_{1}(\hat{t})=\omega_{0}^{2} u_{2}+\omega_{0}^{2} \mu u_{2}\left(\hat{t}+\tau_{1}\right), \quad \hat{t}=-t, \\
& \dot{u}_{2}(\hat{t})=-u_{1}+2 \delta_{0} \omega_{0} u_{2}+\omega_{0}^{2} \mu \delta_{1} u_{2}\left(\hat{t}+\tau_{2}\right),
\end{aligned}
$$

in $\hat{C}:=\hat{C}\left([0, \tau], \mathfrak{R}^{2}\right)$ with respect to the bilinear relation

$$
\begin{aligned}
\left(\psi_{j}(s), \phi_{k}(\theta)\right)= & \psi_{j}(0) \phi_{k}(0)-\mu \delta_{1} \omega_{0}^{2}\left\{\psi_{j}(0) \phi_{k}\left(-\tau_{1}\right)\right\} \\
& -\mu \delta_{1} \omega_{0}^{2} \int_{-\tau_{2}}^{0}\left(\frac{d \psi_{j}\left(\zeta+\tau_{2}\right)}{d \zeta}\right) \phi_{k}(\zeta) d \zeta \\
& +\mu \omega_{0}^{2} \int_{-\tau_{1}}^{0} \psi_{j}\left(\zeta+\tau_{1}\right) \phi_{k}(\zeta) d \zeta, \quad j, k=1,2
\end{aligned}
$$

where $\phi_{k}(\theta) \in C$ and $\psi_{j}(s) \in \hat{C}$. Solutions to the linear DDEs are identical. However, the only difference is that each set of solutions is described in a separate space. That is, solutions of (3.1) are contained in $C$, while those of (3.3) are contained in the adjoint space $\hat{C}$.

We assume that $\lambda_{1,2}=v(\mu) \pm i \omega(\mu), v(\mu)>0, \omega(\mu) \neq 0$ are solutions to (3.2) satisfying $v\left(\mu_{c}\right)=0, \omega\left(\mu_{c}\right) \neq 0, \mathfrak{R} e\{d \Delta(\lambda, \mu) / d \mu\} \neq 0$ at Hopf bifurcation and all other solutions of $\Delta(\lambda, \mu)=0$ correspond to eigenvalues with negative real parts. When we put $\lambda_{1}=i \omega$ into (3.2) and set the resulting real and imaginary parts to zero, we obtain

$$
\begin{gathered}
\omega^{2}-\mu \omega_{0}^{2}\left(\delta_{1} \sin \omega \tau_{2}+\omega^{-1} \cos \omega \tau_{1}\right) \omega-\omega_{0}^{2}=0, \\
\left(2 \delta_{0}+\mu \omega_{0} \delta_{1} \cos \omega \tau_{2}\right) \omega_{0} \omega-\omega_{0}^{2} \mu \sin \omega \tau_{1}=0 .
\end{gathered}
$$

These are the explicit expressions for the determination of boundaries of stable and unstable solutions in the phase plane, say $\left(\mu_{1} \delta_{0}\right)$. By the implicit function theorem, we take the derivative of $\Delta(\lambda, \mu)=0$ with respect to $\mu$. Then the substitution of $\lambda_{1}=i \omega$ into the resulting equations yields the expression for $\{d \Delta(\lambda, \mu) / d \mu\}_{\lambda=i \omega, \mu=\mu_{c}}$, whose real part $\mathfrak{R} e\{d \Delta(\lambda, \mu) / d \mu\}_{\lambda=i \omega, \mu=\mu_{c}}$ is a nonzero quantity.

We decompose $C$ by all the eigenvalues of $\Delta(\lambda, \mu)=0$ as $C=P \oplus Q$, where the eigenvalues $\lambda_{1,2}=v(\mu) \pm i \omega(\mu)$ are associated with the two-dimensional subspace $P$ and the infinite-dimensional complementary subspace $Q$ is associated with all the remaining eigenvalues of $\Delta(\lambda, \mu)=0$. For $\lambda_{1}=i \omega(\mu)$, we have the exponential solutions $\Phi(\theta)=$ $\left[\phi_{1}(\theta), \phi_{2}(\theta)\right], \phi_{1}(\theta)=[\cos \omega \theta, \sin \omega \theta]^{T}$, and $\phi_{2}(\theta)=[-\sin \omega \theta, \cos \omega \theta]^{T}$, which form a basis for $P \in C$ as well as for all the solutions to (3.1). That is, for some constant vector $b$, we have the required initial function $\phi(\theta)=\Phi(\theta) b \in C$. Furthermore, we have $B=\left[[0, \omega]^{T},[-\omega, 0]^{T}\right]$ and it can be shown that $\Phi(\theta)=\Phi(0) e^{B \theta},-\tau \leq \theta \leq 0$. $T$ stands for transpose. The basis for $Q$ is determined by means of the basis function 
$\Phi(\theta)$ for $P \in C$ and the exponential solutions $\Psi(s)=\left[\psi_{1}(s), \psi_{2}(s)\right] \in \hat{C}$, which is the basis for the subspace $\hat{P} \in \hat{C}$ of the adjoint equation (3.3). Again for $\lambda_{1}=i \omega(\mu)$, we have the elements $\psi_{1}(s)=[\cos \omega s,-\sin \omega s]^{T}, \psi_{2}(s)=[\sin \omega s, \cos \omega s]^{T}, 0 \leq s \leq \tau$. The elements of $\Phi(\theta) \in C$, and $\Psi(s) \in \hat{C}$ form the inner product matrix $(\Psi(s), \Phi(\theta))$, namely $(\Psi(s), \Phi(\theta))=\left[\left[\left(\psi_{1}(s), \phi_{1}(\theta)\right),\left(\psi_{2}(s), \phi_{1}(\theta)\right)\right]^{T},\left[\left(\psi_{1}(s), \phi_{2}(\theta)\right),\left(\psi_{2}(s)\right.\right.\right.$, $\left.\left.\phi_{2}(\theta)\right]^{T}\right]$. When we make the substitution of the elements of $(\Psi(s), \Phi(\theta))$ into (3.4), it produces the nonsingular matrix $(\Psi, \Phi)_{n s g}=\left[\left[\psi_{11}, \psi_{21}\right]^{T},\left[-\psi_{12}, \psi_{22}\right]^{T}\right]$ where

$$
\begin{aligned}
& \psi_{11}=\left(1-\omega_{0}^{2} \tau_{1} \mu \cos \omega \tau_{1}\right)-\delta_{1} \omega_{0}^{2} \mu\left(\cos \omega \tau_{2}-\omega \tau_{2} \sin \omega \tau_{2}\right), \quad \psi_{22}=\psi_{11}, \\
& \psi_{12}=-\delta_{1} \omega_{0}^{2} \mu\left(\omega \tau_{2} \cos \omega \tau_{2}+\sin \omega \tau_{2}\right)-\omega_{0}^{2} \tau_{1} \mu \sin \omega \tau_{1}, \quad \psi_{21}=-\psi_{12} .
\end{aligned}
$$

With this nonsingular matrix, we know that the basis $\Psi(s) \in \hat{C}$ for $\hat{P}$ can be normalized to a new basis $\bar{\Psi}(s)=\left[\bar{\psi}_{1}(s), \bar{\psi}_{2}(s)\right] \in \hat{C}$, where its elements $\bar{\psi}_{1}(s)=\left[\bar{\psi}_{11}(s), \bar{\psi}_{21}(s)\right]^{T}$, $\bar{\psi}_{2}(s)=\left[\bar{\psi}_{12}(s), \bar{\psi}_{22}(s)\right]^{T}$ are obtained by evaluating $\bar{\Psi}(s)=(\Psi, \Phi)_{n s g}^{-1} \Psi(s)$. Namely,

$$
\begin{aligned}
& \bar{\psi}_{11}(s)=\left(\psi_{11}^{2}+\psi_{12}^{2}\right)^{-1}\left(\psi_{22} \cos \omega s+\psi_{12} \sin \omega s\right), \\
& \bar{\psi}_{12}(s)=\left(\psi_{11}^{2}+\psi_{12}^{2}\right)^{-1}\left(\psi_{22} \sin \omega s-\psi_{12} \cos \omega s\right), \\
& \bar{\psi}_{21}(s)=-\left(\psi_{11}^{2}+\psi_{12}^{2}\right)^{-1}\left(\psi_{21} \cos \omega s+\psi_{11} \sin \omega s\right), \\
& \bar{\psi}_{22}(s)=-\left(\psi_{11}^{2}+\psi_{12}^{2}\right)^{-1}\left(\psi_{21} \sin \omega s-\psi_{11} \cos \omega s\right) .
\end{aligned}
$$

Then the substitution of the new elements $\left(\bar{\psi}_{j}(s), \phi_{k}(\theta)\right), j, k=1,2$ of the inner product matrix $(\bar{\Psi}(s), \Phi(\theta))$ into (3.4) yields the identity matrix $(\Psi, \Phi)_{\text {id }}=I$. Consequently on the subspaces $P, Q \in C$, we have the characterizations:

$$
\begin{aligned}
& P \equiv\left\{\phi(\theta) \in C \mid \phi(\theta)=\phi^{P}(\theta)+\phi^{Q}(\theta), \phi^{P}(\theta)=\Phi(\theta) b, b:=\left(\bar{\Psi}(s), \phi^{P}(\theta)\right)\right\}, \\
& Q=\left\{\phi(\theta) \in C, \phi^{Q}(\theta)=\phi(\theta)-\phi^{P}(\theta),(\bar{\Psi}(s), \phi(\theta))=0\right\} .
\end{aligned}
$$

Then on $P$ the transformation $x_{t}^{P}(\phi(\theta), \mu)=\Phi(\theta) z(t), z(t)=(\bar{\Psi}(s), \phi(\theta)), z(t) \in$ $\mathfrak{R}^{2}$ will give rise to the following relationships: $x_{1}(t)=z_{1}(t), x_{2}(t)=z_{2}(t), \theta=0$, $x_{1}(t-\tau)=z_{1} \cos \omega \tau+z_{2} \sin \omega \tau, z_{2}(t-\tau)=-z_{1} \sin \omega \tau+z_{2} \cos \omega \tau, \theta=-\tau$.

Therefore, with the above characterizations, specifically the bases $\Phi(\theta) \in C, \bar{\Psi}(s) \in$ $\hat{C}$ so that $(\Psi, \Phi)_{\text {id }}=I$, the matrix $B=\left[[0, \omega]^{T},[-\omega, 0]^{T}\right]$ and the coefficients of the matrix $\bar{\Psi}(0)$ of the normalized basis $\bar{\Psi}(s)$ for $\hat{P} \in \hat{C}$ of the adjoint equation (3.3), we have the equivalent stochastic ODEs on the centre manifold $M_{\mu} \in C\left([-\tau, 0], \mathfrak{R}^{2}\right)$

$$
\begin{gathered}
\dot{z}_{1}(t)=-\omega z_{2}-\varepsilon\left\{\bar{\psi}_{12}(0)\left\{\tilde{\mu} \omega_{0}^{2}\left(z_{1} \cos \omega \tau_{1}+z_{2} \sin \omega \tau_{1}\right)+\varepsilon^{-1 / 2} \sigma_{0} \xi(t) z_{2}\right\}\right. \\
\left.-\bar{\psi}_{11}(0) \tilde{\mu} \omega \omega_{0}^{2} \delta_{1}\left(z_{2} \cos \omega \tau_{2}-z_{1} \sin \omega \tau_{2}\right)\right\}, \\
\dot{z}_{2}(t)=\omega z_{1}-\varepsilon\left\{\bar{\psi}_{22}(0)\left\{\tilde{\mu} \omega_{0}^{2}\left(z_{1} \cos \omega \tau_{1}+z_{2} \sin \omega \tau_{1}\right)+\varepsilon^{-1 / 2} \sigma_{0} \xi(t) z_{2}\right\}\right. \\
\left.-\bar{\psi}_{21}(0) \tilde{\mu} \omega \omega_{0}^{2} \delta_{1}\left(z_{2} \cos \omega \tau_{2}-z_{1} \sin \omega \tau_{2}\right)\right\} .
\end{gathered}
$$


The polar coordinate transformation $z_{1}=a \sin \Theta, z_{2}=-a \cos \Theta, \Theta=\omega t+\varphi$ writes (3.9) in terms of amplitude $a$ and phase $\varphi$ relations. Then the method according to Has'minskiǐ [5] and Stratonovich [9] produces the uncoupled averaged equations

$$
\begin{aligned}
d a= & -\frac{1}{2} a \varepsilon\left\{\omega_{0}^{2} \tilde{\mu}\left(\kappa_{111}+\delta_{1} \kappa_{112}\right)-\frac{1}{4} \sigma_{0}^{2}\left(\bar{\psi}_{12}^{2}(0)+\bar{\psi}_{22}^{2}(0)\right)\right\} d t \\
& +\varepsilon^{1 / 2} \sqrt{\frac{\bar{\psi}_{12}^{2}(0)+\bar{\psi}_{22}^{2}(0)}{8}} \sigma_{0} a d W_{1}(t), \\
d \varphi= & -\frac{1}{2} \varepsilon\left\{\omega_{0}^{2} \tilde{\mu}\left(\kappa_{113}+\delta_{1} \kappa_{114}\right)\right\} d t+\varepsilon^{1 / 2} \sqrt{\frac{\bar{\psi}_{12}^{2}(0)+\bar{\psi}_{22}^{2}(0)}{8}} \sigma_{0} d W_{2}(t), \\
\kappa_{111}= & \bar{\psi}_{12}(0) \cos \omega \tau_{1}+\bar{\psi}_{22}(0) \sin \omega \tau_{1}, \quad \kappa_{112}=\bar{\psi}_{11}(0) \sin \omega \tau_{2}-\bar{\psi}_{21}(0) \cos \omega \tau_{2}, \\
\kappa_{113}= & \bar{\psi}_{22}(0) \cos \omega \tau_{1}-\bar{\psi}_{12}(0) \sin \omega \tau_{1}, \quad \kappa_{114}=\bar{\psi}_{11}(0) \cos \omega \tau_{2}+\bar{\psi}_{21}(0) \sin \omega \tau_{2} .
\end{aligned}
$$

We set $\rho=\log |a|$ to get $d \rho(t)=(1 / a) d a$, whose direct integration yields with probability one (w.p.1) the Lyapunov exponent

$$
\pi_{\exp }:=-\left(\frac{1}{2}\right) \varepsilon\left\{\omega_{0}^{2} \tilde{\mu}\left(\kappa_{111}+\delta_{1} \kappa_{112}\right)-\left(\frac{1}{4}\right) \sigma_{0}^{2}\left(\bar{\psi}_{12}^{2}(0)+\bar{\psi}_{22}^{2}(0)\right)\right\} .
$$

Thus, the solution response is asymptotically stable w.p.1, if for $\pi_{\exp }=0$, we have $\omega_{0}^{2} \tilde{\mu}\left(\kappa_{111}+\delta_{1} \kappa_{112}\right)>(1 / 4) \sigma_{0}^{2}\left(\bar{\psi}_{12}^{2}(0)+\bar{\psi}_{22}^{2}(0)\right)$, otherwise unstable when this inequality fails.

In order to obtain the $p$ th-moment exponent $\mathfrak{I}(p)$ for the amplitude equations (3.10), we set up an Itô equation for $a^{p}$ as follows:

$$
\begin{aligned}
d\left(a^{p}\right)= & -\frac{1}{2} p \varepsilon\left\{\omega_{0}^{2} \tilde{\mu}\left(\kappa_{111}+\delta_{1} \kappa_{112}\right)-\frac{1}{4} \sigma_{0}^{2} p\left(\bar{\psi}_{12}^{2}(0)+\bar{\psi}_{22}^{2}(0)\right)\right\} a^{p} d t \\
& +\varepsilon^{1 / 2} \sqrt{\frac{\bar{\psi}_{12}^{2}(0)+\bar{\psi}_{22}^{2}(0)}{8}} \sigma_{0} a^{p} p d W_{1}(t) .
\end{aligned}
$$

From which and taking the expectation of both sides of (3.12) leads to $\mathfrak{T}(p):=$ $-(1 / 2) p \varepsilon\left\{\omega_{0}^{2} \tilde{\mu}\left(\kappa_{111}+\delta_{1} \kappa_{112}\right)-(1 / 4) \sigma_{0}^{2} p\left(\bar{\psi}_{12}^{2}(0)+\bar{\psi}_{22}^{2}(0)\right)\right\}$. Putting $\mathfrak{J}(p)=\{0\}_{p=p_{1}}$ produces two values of the stability index $p$, and thus we can say that the solution response is $p$ th stable if and only if the inequality $0<p_{1}<4 \omega_{0}^{2} \tilde{\mu}\left(\kappa_{111}+\delta_{1} \kappa_{112}\right) /$ $\sigma_{0}^{2}\left(\bar{\psi}_{12}^{2}(0)+\bar{\psi}_{22}^{2}(0)\right)$ holds.

3.1. An illustrative example II. The reduction of the infinite-dimensional character of DDEs to ODEs is further demonstrated by considering the nonlinear delay equation

$$
\begin{aligned}
\dot{x}_{1}(t)= & x_{2}, \\
\dot{x}_{2}(t)= & -\omega_{0}^{2}\left\{(1-\mu) x_{1}+\mu x_{1}(t-\tau)\right\}-2 \delta_{0} \omega_{0} x_{2} \\
& -\varepsilon \omega_{0}^{2}\left\{\beta_{3} \mu x_{1}^{3}(t-\tau)+\sigma_{3} x_{1}^{3}\right\},
\end{aligned}
$$

which is precisely the classical Duffing equation when $\mu=0$. The parameters $\beta_{3}, \sigma_{3}$ in these equations denote the coefficients of the nonlinearity. The linearized part of (3.13)

$$
\dot{x}_{1}(t)=x_{2}, \quad \dot{x}_{2}(t)=-\omega_{0}^{2}\left\{(1-\mu) x_{1}+\mu x_{1}(t-\tau)\right\}-2 \delta_{0} \omega_{0} x_{2},
$$


in $C:=C\left([-\tau, 0], \mathfrak{R}^{2}\right)$ has the adjoint form

$$
\dot{u}_{1}(\hat{t})=\omega_{0}^{2}\left\{(1-\mu) u_{2}+\mu u_{2}(\hat{t}+\tau)\right\}, \quad \dot{u}_{2}(\hat{t})=-u_{1}+2 \delta_{0} \omega_{0} u_{2},
$$

in $\hat{C}:=\hat{C}\left([0, \tau], \mathfrak{R}^{2}\right)$ and the bilinear relation

$$
\left(\psi_{j}(s), \phi_{k}(\theta)\right)=\psi_{j}(0) \phi_{k}(0)+\omega_{0}^{2} \mu \int_{-\tau}^{0} \psi_{j}(\tau+\zeta) \phi_{k}(\zeta) d \zeta, \quad j, k=1,2 .
$$

Furthermore, (3.14) has the characteristic equation

$$
\Delta(\lambda, \mu):=\lambda^{2}+2 \zeta_{0} \omega_{0} \lambda+\omega_{0}^{2}\left\{(1-\mu)+\mu e^{-\lambda \tau}\right\}=0,
$$

which may have eigenvalues of the form $\lambda_{1,2}= \pm i \omega(\mu)$ for $\mu=\mu_{c}>0$, and while all the remaining ones can be assumed to have negative real parts. Substituting $\lambda_{1}=i \omega$ into (3.17), we get the expressions for stability characterization $\omega^{2}-\omega_{0}^{2}\{(1-\mu)+$ $\mu \cos \omega \tau\}=0$ and $2 \delta_{0} \omega_{0} \omega-\omega_{0}^{2} \mu \sin \omega \tau$.

Next for the eigenvalues of $\lambda_{1,2}= \pm i \omega(\mu)$, we define the bases of $P \in C$ and $\hat{P} \in \hat{C}$ of the linear delay equations (3.14), (3.15), and (3.16), namely $\Phi(\theta)=\left[\phi_{1}(\theta), \phi_{2}(\theta)\right] \in C$, $\phi_{1}(\theta)=[\cos \omega \theta, \sin \omega \theta]^{T}, \phi_{2}(\theta)=[-\sin \omega \theta, \cos \omega \theta]^{T}$, and $\Psi(s)=\left[\psi_{1}(s), \psi_{2}(s)\right] \in$ $\hat{C}, \psi_{1}(s)=[\cos \omega s,-\sin \omega s]^{T}, \psi_{2}(s)=[\sin \omega s, \cos \omega s]^{T}$. The computation of the inner product matrix $(\Psi(s), \Phi(\theta))=\left(\psi_{j}(s), \phi_{k}(\theta)\right), j, k=1,2$, yields $(\Psi, \Phi)_{n s g}=\left[\left[\psi_{11}\right.\right.$, $\left.\left.\psi_{21}\right]^{T},\left[-\psi_{12}, \psi_{22}\right]^{T}\right]$ where $\psi_{11}=\psi_{22}=1+\omega_{0}^{2} \mu \tau \cos \omega \tau, \psi_{12}=\omega_{0}^{2} \mu \tau \sin \omega \tau$, and $\psi_{21}=-\psi_{12}$. We define the new basis $\bar{\Psi}(s) \in \hat{C}$ for $\hat{P}$ by computing $\bar{\Psi}(s)=(\Psi, \Phi)_{n s g}^{-1} \Psi(s)$, which yields

$$
\begin{aligned}
\bar{\Psi}(s) & =\left[\bar{\psi}_{1}(s), \bar{\psi}_{2}(s)\right]=\left[\begin{array}{ll}
\bar{\psi}_{11}(s) & \bar{\psi}_{12}(s) \\
\bar{\psi}_{21}(s) & \bar{\psi}_{22}(s)
\end{array}\right], \quad 0 \leq s \leq \tau, \\
\bar{\psi}_{11}(s) & =\left(\psi_{11}^{2}+\psi_{12}^{2}\right)^{-1}\left(\psi_{22} \cos \omega s+\psi_{12} \sin \omega s\right), \\
\bar{\psi}_{12}(s) & =\left(\psi_{11}^{2}+\psi_{12}^{2}\right)^{-1}\left(\psi_{22} \sin \omega s-\psi_{12} \cos \omega s\right), \\
\bar{\psi}_{21}(s) & =-\left(\psi_{11}^{2}+\psi_{12}^{2}\right)^{-1}\left(\psi_{21} \cos \omega s+\psi_{11} \sin \omega s\right), \\
\bar{\psi}_{22}(s) & =-\left(\psi_{11}^{2}+\psi_{12}^{2}\right)^{-1}\left(\psi_{21} \sin \omega s-\psi_{11} \cos \omega s\right) .
\end{aligned}
$$

Again the substitution of the elements $\left(\bar{\psi}_{j}(s), \phi_{k}(\theta)\right), j, k=1,2$, of the new inner product $(\bar{\Psi}(s), \Phi(\theta))$ into (3.16) produces the $2 \times 2$ identity matrix $(\Psi, \Phi)_{\text {id }}=I$. Since the elements $\phi(\theta), X_{0}(\theta)$ are in $C$, we have the corresponding projections onto the subspaces $P, Q \in C$ as follows:

$$
\begin{gathered}
P=\left\{\phi(\theta) \in C, \phi^{P}(\theta)=\phi(\theta)-\phi^{Q}(\theta) \mid \phi^{P}(\theta)=\Phi(\theta) b,\right. \\
\left.b=\left(\bar{\Psi}(s), \phi^{P}(\theta)\right), X_{0}^{P}(\theta):=\Phi(\theta) \bar{\Psi}(0)\right\}
\end{gathered}
$$

and

$$
Q=\phi(\theta) \in C, \quad \phi^{Q}(\theta)=\phi(\theta)-\phi^{P}(\theta) \mid\left(\bar{\Psi}(s), \phi^{Q}(\theta)\right)=0 .
$$


Consequently, the change of variable $x_{t}^{P}(\theta)=\Phi(\theta) z(t), z(t) \in \mathfrak{R}^{2}, z(t)=(\bar{\Psi}(s)$, $\phi^{P}(\theta)$ ) produces the set of nonlinear ODEs on the centre manifold $M_{\mu} \in C\left([-\tau, 0], \mathfrak{R}^{2}\right)$

$$
\begin{aligned}
\dot{z}_{1}(t)=-\omega z_{2}+\varepsilon \bar{\psi}_{12}(0)\{ & \beta_{3} \mu_{c}\left(z_{1} \cos \omega \tau+z_{2} \sin \omega \tau\right)^{3}+\sigma_{3} z_{1}^{3} \\
& \left.+\omega_{0}^{2} \tilde{\mu}\left(z_{1}-\left(z_{1} \cos \omega \tau+z_{2} \sin \omega \tau\right)\right)\right\}, \\
\dot{z}_{2}(t)=\omega z_{1}+\varepsilon \bar{\psi}_{22}(0)\{ & \beta_{3} \mu_{c}\left(z_{1} \cos \omega \tau+z_{2} \sin \omega \tau\right)^{3}+\sigma_{3} z_{1}^{3} \\
+ & \left.\omega_{0}^{2} \tilde{\mu}\left(z_{1}-\left(z_{1} \cos \omega \tau+z_{2} \sin \omega \tau\right)\right)\right\},
\end{aligned}
$$

where $\bar{\psi}_{12}(0)=-\left(\psi_{11}^{2}+\psi_{12}^{2}\right)^{-1} \psi_{12}$ and $\bar{\psi}_{22}(0)=\left(\psi_{11}^{2}+\psi_{12}^{2}\right)^{-1} \psi_{11}$. Writing the ODEs (3.21) into amplitude and phase relations at the expense of the transformation $z_{1}=$ $a \sin \Theta, z_{2}=-a \cos \Theta, \Theta=\omega t+\varphi$, and then applying the integral averaging method leads to the uncoupled averaged equations

$$
\begin{aligned}
\dot{a}(t) & =-\frac{\varepsilon a}{8}\left\{3 q_{311} a^{2}+4 q_{111} \tilde{\mu}\right\}, \\
\dot{\varphi}(t) & =-\frac{\varepsilon}{8}\left\{3 p_{311} a^{2}+4 p_{111} \tilde{\mu}\right\}, \\
q_{311} & =\beta_{3} \mu_{c}\left\{\bar{\psi}_{12}(0) \cos \omega \tau+\bar{\psi}_{22}(0) \sin \omega \tau\right\}+\sigma_{3} \bar{\psi}_{12}(0), \\
q_{111} & =\omega_{0}^{2}\left\{\bar{\psi}_{12}(0)(1-\cos \omega \tau)-\bar{\psi}_{22}(0) \sin \omega \tau\right\}, \\
p_{311} & =\beta_{3} \mu_{c}\left\{\bar{\psi}_{22}(0) \cos \omega \tau-\bar{\psi}_{12}(0) \sin \omega \tau\right\}+\sigma_{3} \bar{\psi}_{22}(0), \\
p_{111} & =\omega_{0}^{2}\left\{\bar{\psi}_{22}(0)(1-\cos \omega \tau)-\bar{\psi}_{12}(0) \sin \omega \tau\right\} .
\end{aligned}
$$

By the relationship $a=a_{0}+\rho$, we have the variational equation

$$
\dot{\rho}(t)=-\left(\frac{\varepsilon}{8}\right)\left\{9 q_{311} a_{0}^{2}+4 q_{111} \tilde{\mu}\right\} \rho,
$$

where for $\dot{\rho}(t)=0$ yields the scalar equation

$$
g\left(a_{0}, \mu\right):=-\left(\frac{\varepsilon}{8}\right)\left\{9 a_{0}^{2} q_{311}+4 q_{111} \tilde{\mu}\right\}=0
$$

where $a_{0}$ is the steady state value determined by setting $\dot{a}(t)=\dot{\varphi}(t)=0$. Thus, it can be shown that the solution responses of the nonlinear delay equations (3.13) show the subcritical stability for $9 q_{311}<-4 q_{111}$ and $4 q_{111}>0$, and supercritical stability when $9 q_{311}>4 q_{111}$ and $4 q_{111}>0$.

4. Conclusion. An attempt to establish a unified framework for the study of stability of second-order differential equations with multiple and distinct time delays in the displacement and derivative functions, plus a derivative process of the damping coefficient, has been made. Andronov-Hopf bifurcation, centre manifold theorem, the integral stochastic averaging method, and $p$ th-moment Lyapunov exponents have been employed in the development of the framework. Sufficient conditions for stability in the deterministic and stochastic sense have been presented. It is felt that this framework will uncover a wealth of phenomena of stochastic dynamical systems with delays since the investigations are conducted in the appropriate infinite-dimensional space $C\left([-\tau, 0], \mathfrak{R}^{2}\right)$ without the assumption of small delay. 


\section{REFERENCES}

[1] L. Arnold and W. Kliemann, Large Deviations of Linear Stochastic Differential Equations, Lecture Notes in Control and Information Science, vol. 96, Springer-Verlag, New York, 1987.

[2] L. Arnold, E. Oeljeklaus, and É. Pardoux, Almost sure and moment stability for linear Itô equations, Lyapunov Exponents (Bremen, 1984), Lecture Notes in Mathematics, vol. 1186, Springer, Berlin, 1986, pp. 129-159.

[3] P. H. Baxendale, Invariant measures for nonlinear stochastic differential equations, Lyapunov Exponents (Oberwolfach, 1990) (L. Arnold, H. Crauel, and J. P. Ekmann, eds.), Lecture Notes in Mathematics, vol. 1486, Springer, Berlin, 1991, pp. 123-140.

[4] J. Hale, Theory of Functional Differential Equations, Springer-Verlag, New York, 1977.

[5] R. Z. Has'minskiĭ, Stochastic processes defined by differential equations with a small parameter, Teor. Verojatnost. i Primenen 11 (1966), 240-259.

[6] Bogoliubov N. N. and Y. A. Mitropolsky, Asymptotic Methods in the Theory of Nonlinear Oscillations, Gordon and Breach, New York, 1960.

[7] V. I. Oseledec, A multiplicative ergodic theorem. Characteristic Ljapunov, exponents of dynamical systems, Trudy Moskov. Mat. Obšč. 19 (1968), 179-210.

[8] T. T. Soong, Active Structural Control: Theory and Practice, Longman Science \& TechnicalJohn Wiley, New York, 1990.

[9] R. L. Stratonovich, Topics in the Theory of Random Noise II, Gordon and Breach, New York, 1967.

M. S. FofANA: NONLINEAR MANUFACTURING SYSTEMS LABORATORY, MANUFACTURING ENGINeERing Program, Worcester Polytechnic Institute, Worcester, MA 01609-2280, USA

E-mail address: ms fofana@wpi . edu 


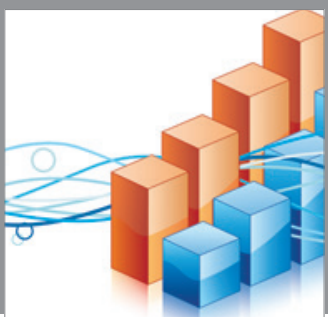

Advances in

Operations Research

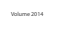

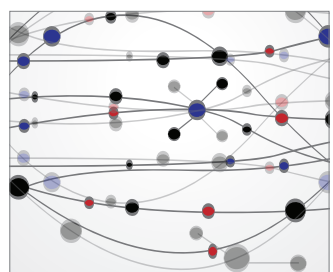

\section{The Scientific} World Journal
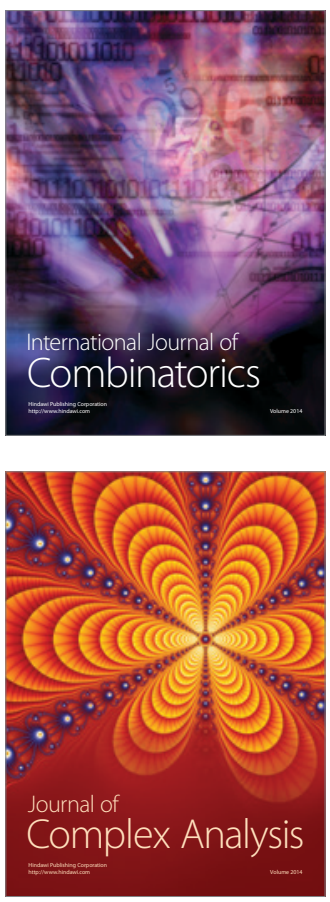

International Journal of

Mathematics and

Mathematical

Sciences
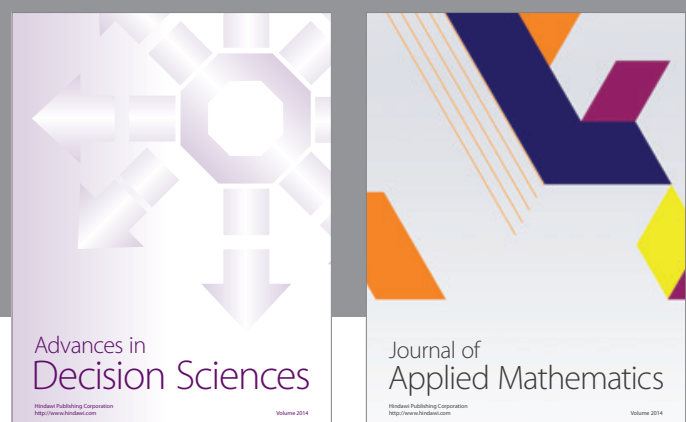

Journal of

Applied Mathematics
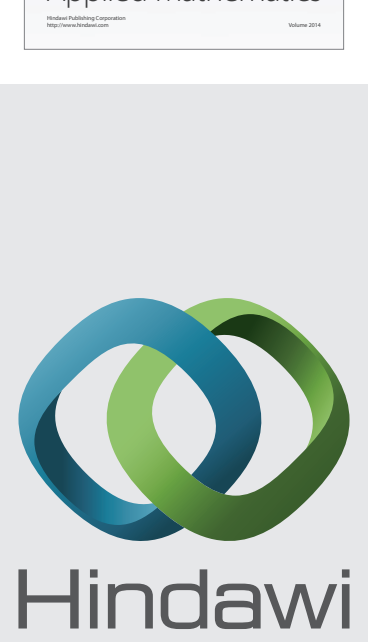

Submit your manuscripts at http://www.hindawi.com
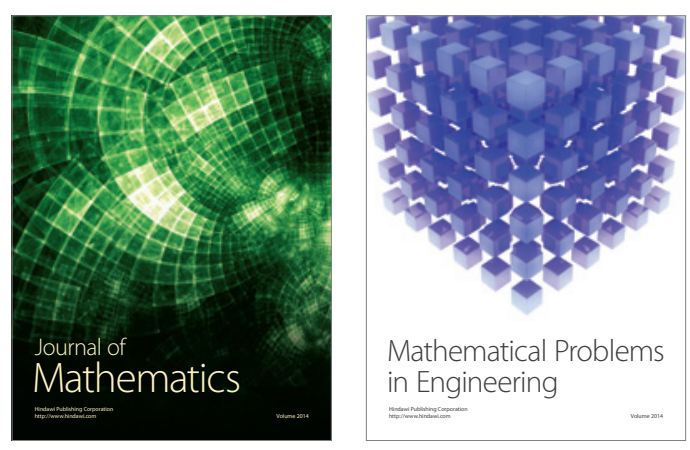

Mathematical Problems in Engineering
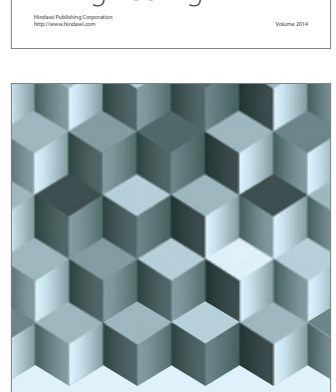

Journal of

Function Spaces
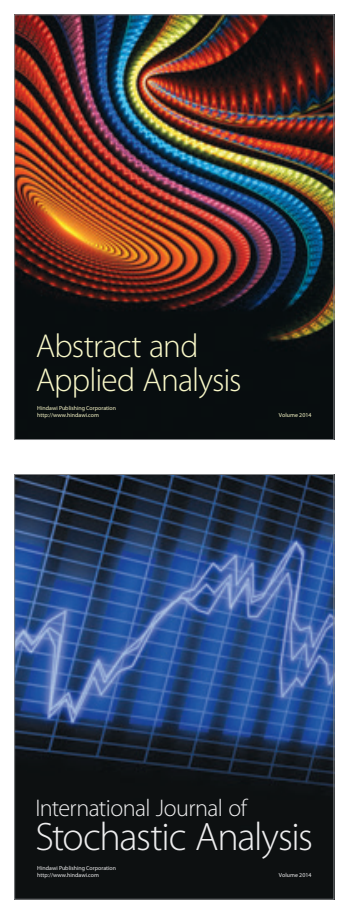

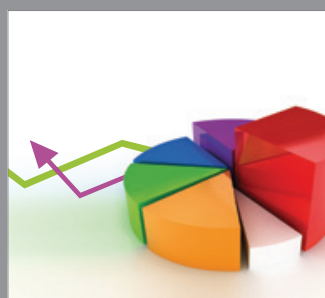

ournal of

Probability and Statistics

Promensencen
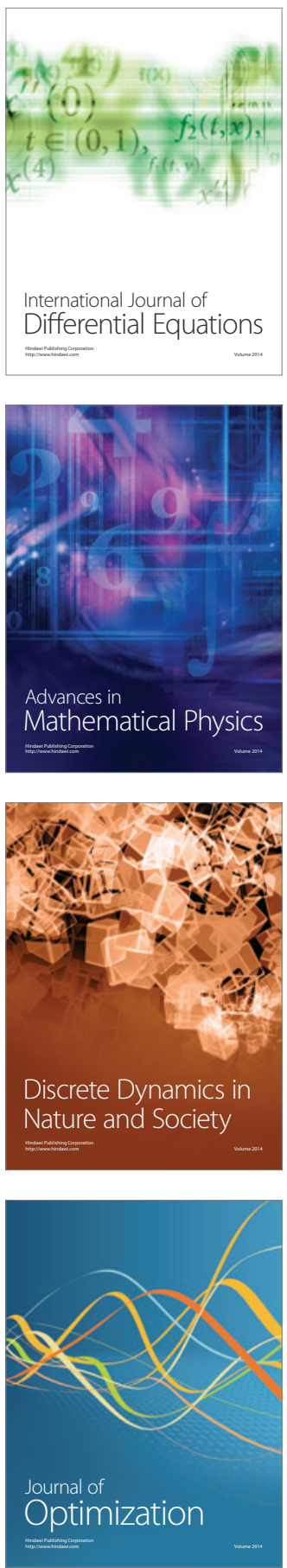